\title{
TORIC DELIGNE-MUMFORD STACKS AND THE BETTER BEHAVED VERSION OF THE GKZ HYPERGEOMETRIC SYSTEM
}

\author{
R. PAUL HORJA
}

\begin{abstract}
We generalize the combinatorial description of the orbifold (Chen-Ruan) cohomology and of the Grothendieck ring of a Deligne-Mumford toric stack and its associated stacky fan in a lattice $N$ in the presence of a deformation parameter $\beta \in N \otimes \mathbb{C}$. As an application, we construct a topological mirror symmetry map that produces a complete system of $\Gamma$-series solutions to the better behaved version of the GKZ hypergeometric system for $\beta \in N \otimes \mathbb{C}$.
\end{abstract}

\section{INTRODUCTION}

This note is a continuation of some recent joint work of the author with Lev Borisov [4] on the better behaved version of the GelfandKapranov-Zelevinsky hypergeometric system. The first two sections after the introduction, which may be of independent interest, contain a construction of certain modules over the Stanley-Reisner (SR) cohomology and describe their Grothendieck ring interpretation in the context of toric Deligne-Mumford stacks. The notion of SR-cohomology and the computation of the Grothendieck ring of a smooth DeligneMumford stack have been discussed in [5]; when the stack is projective, the SR-cohomology is the same as the orbifold (Chen-Ruan) cohomology. For a given stacky fan $\Sigma$ in a lattice $N$ and a parameter $\beta \in N \otimes \mathbb{C}$, we give a combinatorial description (cf. Definition 2.7) of the deformed SR-cohomology module $H \mathbb{Z}[\boldsymbol{\Sigma} ; \beta]$, and of the deformed Grothendieck ring $K \mathbb{C}[\Sigma ; \beta]$ (cf. Definition 3.1). Not surprisingly, the two deformed notions are related by a Chern character-type map (Theorem 3.3) and, in the case of an integral parameter $\beta \in N$, we recover the geometric calculations of SR-cohomology and of the Grothendieck ring of a toric Deligne-Mumford stack, respectively. Moreover, Proposition 2.5 shows that it is possible to reduce the discussion for a complex parameter $\beta \in N \otimes \mathbb{C}$ to the more geometric case of the real parameter $\Re \beta+\delta \Im \beta \in N \otimes \mathbb{R}$ for all sufficiently small real numbers $\delta>0$. It is tempting to conjecture that the constructions presented here and

The author was partially supported by the NSA grant MDA904-10-1-0190. 
the presence of certain "walls" in the space of parameters $\beta \in N \otimes \mathbb{R}$ (cf. Example 3.5) have an equivariant algebra-geometric interpretation, but we postpone the discussion of such speculations for future work.

As an application, we present a construction of a complete set of $\Gamma$ series solutions to the better behaved GKZ system in the case $\beta \in$ $N \otimes \mathbb{C}$. For the classical GKZ system, such solutions have been obtained in various degrees of generality in the works of [8], [11], [6], as well as in the book [10].

In a celebrated paper, Gelfand, Kapranov and Zelevinsky [7] considered a system of linear partial differential equations associated to the set $\mathcal{A}$ and any parameter $\beta \in N \otimes \mathbb{C}$. Besides being an interesting object to study in itself, the GKZ system has become a fundamental tool in mirror symmetry after the discovery by [1 that the PicardFuchs equations associated to the periods of Calabi-Yau hypersurfaces in toric varieties are in fact of GKZ type. If the toric ideal associated to $\mathcal{A}$ is not Cohen-Macaulay, there are non-generic values of $\beta$ for which the rank of the solution set jumps. A thorough analysis of this property is contained in the work 9. However, this phenomenon brings with it a degree of non-functoriality in some of the applications of the GKZ system to the more recent categorical questions in algebraic geometry and homological mirror symmetry.

This discrepancy constituted the starting point of our recent joint work [4], where we proposed a better behaved version of the GKZ system whose space of solutions has the expected number of solutions in all cases. We framed the definition in a context where the lattice is replaced by a finitely generated abelian group $N$. and the set $\mathcal{A}$ determining the PDE system is replaced by an $n$-tuple of elements of $N$, with possible repetitions.

In section 4 of this paper, we only consider the case when $N$ is a lattice of rank $d$, and $\mathcal{A}=\left\{v_{1}, \ldots, v_{k}\right\}$ is a set. We assume that the elements of $\mathcal{A}$ generate the lattice as an abelian group and that there exists a group homomorphism $\operatorname{deg}: N \rightarrow \mathbb{Z} \operatorname{such}$ that $\operatorname{deg}\left(v_{i}\right)=1$ for any element $v_{i} \in \mathcal{A}$. Let $K \subset N \otimes \mathbb{R}$ be the cone over the polytope $\Delta=\operatorname{conv}(\mathcal{A})$, the convex hull of the elements of $\mathcal{A}$.

For any parameter $\beta$ in $N \otimes \mathbb{C}$, the better behaved Gelfand-KapranovZelevinsky system consists of the system of partial differential equations

$$
\partial_{i} \Phi_{v}=\Phi_{v+v_{i}}, \text { for all } v \in K \cap N, i \in\{1, \ldots, k\}
$$

and the linear equations 
(2)

$\sum_{i=1}^{k} g\left(v_{i}\right) x_{i} \partial_{i} \Phi_{v}=g(\beta-v) \Phi_{v}$, for all $g \in M=\operatorname{Hom}(N, \mathbb{Z}), v \in K \cap N$.

A solution to the better behaved GKZ system is then a sequence of functions of $k$ variables $\left(\Phi_{v}\left(x_{1}, \ldots, x_{k}\right)\right)_{v \in K \cap N}$. Alternatively, it can be viewed as a function in $k$ variables

$$
\Phi_{K}\left(x_{1}, \ldots, x_{k}\right):=\sum_{v \in K \cap N} \Phi_{v}\left(x_{1}, \ldots, x_{k}\right)[v]
$$

with values in the completion $\mathbb{C}[K \cap N]^{\wedge}$ of the ring $\mathbb{C}[K \cap N]$. In section 4 of this note, we use the constructions of the first section to obtain $\operatorname{vol}(\Delta)$ linearly independent $\Gamma$-series solutions for the better behaved GKZ system which are analytic in an non-empty open set $U_{\boldsymbol{\Sigma}}$ corresponding to any regular simplicial fan structure supported on $K$ whose one dimensional cones are generated by elements of $\mathcal{A}$. The solutions are obtained with the help of a "topological mirror symmetry map" (cf. Theorem 4.8). In conjunction with the results of [4], this construction gives an explicit complete set of solutions in the general case of a finitely generated abelian group $N$, a collection $\mathcal{A}$ with possible repetitions and any parameter $\beta \in N \otimes \mathbb{C}$.

\section{Deformed modules over the SR-COHomology of A REDUCED TORIC DM STACK}

In this section, $N \simeq \mathbb{Z}^{d}$ is a lattice of rank $d, \Sigma=\left(\Sigma,\left\{v_{i}\right\}_{1 \leq i \leq k}\right)$ is a simplicial stacky fan; we assume that any of the elements $v_{i} \in N$ generates a ray (one dimensional cone) of the rational simplicial fan $\Sigma$.

Notation. As it is customary, we denote by $\{a\}, 0 \leq\{a\}<1$, the fractionary part of the real number $a$. For any cone $\sigma$ of the fan $\Sigma$, we will denote by $I(\sigma) \subset\{1,2, \ldots, k\}$ the subset consisting of those indices $i$ such that $v_{i}$ generates a ray of the cone $\sigma$. To any subset $I \subset\{1,2, \ldots, k\}$, we associate the cone $\sum_{i \in I} \mathbb{R}_{\geq 0} v_{i}$ and we denote it by $\sigma(I)$. We denote by $\sigma$ the cone as well as its support in $N \otimes \mathbb{R}$, and by $\Sigma$ the union of the supports of all the cones of the fan.

For pedagogical reasons, we begin by assuming that $\beta \in N \otimes \mathbb{R}$ is the fixed parameter. For a maximal cone $\sigma$ of $\Sigma$, let $\operatorname{Box}(\sigma ; \beta)$ denote the finite subset of elements $c \in \sigma \subset N \otimes \mathbb{R}$ of the form $n+\beta$, for some 
$n \in N$, such that

$$
c=n+\beta=\sum_{i=1}^{k} \alpha_{i} v_{i},
$$

with $0 \leq \alpha_{i}<1$, for all $i, 1 \leq i \leq k$, and $\alpha_{i}=0$, if $i \notin I(\sigma)$.

Let $\operatorname{Box}(\Sigma ; \beta)$ be the union of the sets $\operatorname{Box}(\sigma ; \beta)$ for all the maximal cones $\sigma$ of $\Sigma$. For any element $c$ in $\operatorname{Box}(\boldsymbol{\Sigma}, \beta)$, the minimal cone of $\Sigma$ containing $c$ is denoted by $\sigma(c)$ and it is called the support cone of $\alpha$. When $\beta \in N$, the set $\operatorname{Box}(\boldsymbol{\Sigma} ; \beta)$ coincides with the usual set $\operatorname{Box}(\boldsymbol{\Sigma}) \subset N$ from the theory of toric DM stacks.

Let $\mathbb{Z}[\boldsymbol{\Sigma}]$ be the deformed semigroup ring defined as the free abelian group with the basis elements [n] for all $n \in N \cap \Sigma$, and the product $[m] \cdot[n]=[m+n]$, when $m$ and $n$ belong to some cone of the fan, and 0 otherwise. This will be the ground ring in our discussion, so we call it $R$.

Definition 2.1. The $R$-module $\mathbb{Z}[\Sigma ; \beta]$ is generated as a free abelian group by the basis elements $[n+\beta]$ for all $n \in N$ such that $n+\beta \in \Sigma$. The module structure is given by the product $\left[n^{\prime}\right] \cdot[n+\beta]:=\left[n^{\prime}+n+\beta\right]$, if there exists a cone of the fan containing $n^{\prime}$ and $n+\beta$, and 0 otherwise.

Remark 2.2. The underlying theme of this section has to do with the change in the structure of the modules $\mathbb{Z}[\boldsymbol{\Sigma} ; \beta]$ when the parameter $\beta$ is allowed to vary in $N \otimes \mathbb{R}$. The module structure is related to the combinatorics of the set $\operatorname{Box}(\boldsymbol{\Sigma} ; \beta)$, and the following example features one of the subtleties that has to be considered.

In the lattice $N=\mathbb{Z}^{2}$ consider the fan $\Sigma$ whose two maximal cones are generated by the pairs of vectors $\left\{v_{1}, v_{2}\right\}$ and $\left\{v_{2}, v_{3}\right\}$, where $v_{1}=$ $(1,0), v_{1}=(1,1), v_{2}=(1,2)$. Let $\beta=(\delta, 0) \in N \otimes \mathbb{R}$, with $0<\delta<1$. We see that $\operatorname{Box}(\boldsymbol{\Sigma} ; \beta)=\{(\delta, 0),(1+\delta, 2)\}$, and that the pointwise limit in $N \otimes \mathbb{R} \simeq \mathbb{R}^{2}$ of $\operatorname{Box}(\boldsymbol{\Sigma} ; \beta)=\{(\delta, 0),(1+\delta, 2)\}$ is the set $\{(0,0),(1,2)\}$. On the other hand, it is certainly the case that $(\delta, 0) \rightarrow$ $(0,0)$ when $\delta \rightarrow 0$, and $\operatorname{Box}(\Sigma ;(0,0))=\{(0,0)\}$. It is possible to gain a general understanding of such a limiting process by defining a $\gamma-$ deformed version of the set $\operatorname{Box}(\boldsymbol{\Sigma} ; \beta)$ for any $\gamma \in N \otimes \mathbb{R}$, but we leave the details of this construction as an exercise. Moreover, a related issue is the subject of Corollary 2.6 which is proved below.

We now move to the more general case $\beta \in N \otimes \mathbb{C}$. We consider a version of the set $\operatorname{Box}(\boldsymbol{\Sigma} ; \beta) \subset N \otimes \mathbb{R} \simeq \mathbb{R}^{d}$ adapted to this situation. Given a maximal cone $\sigma$ of the fan $\Sigma$, let $\operatorname{Bx}(\sigma ; \beta) \subset \mathbb{C}^{k}$ be defined as 
the finite set consisting of elements $\alpha=\left(\alpha_{i}\right)_{1 \leq i \leq k}$ such that

$$
n+\beta=\sum_{i=1}^{k} \alpha_{i} v_{i}
$$

for some $n \in N$, with $0 \leq \Re \alpha_{i}<1$, for all $i, 1 \leq i \leq k$, and $\alpha_{i}=0$, if $i \notin I(\sigma)$. It is clear that, in such a situation, we have that $n+\Re \beta \in \sigma$. The set $\operatorname{Bx}(\Sigma ; \beta) \subset \mathbb{C}^{k}$ is then defined as the union of the sets $\operatorname{Bx}(\sigma ; \beta)$ for all the maximal cones $\sigma$ of $\Sigma$. For any element $\alpha$ in $\operatorname{Bx}(\Sigma, \beta)$, the support cone $\sigma(\alpha)$ of $\alpha$ is now defined as the cone of $\Sigma$ generated by all the $v_{i}$ such that $\alpha_{i} \neq 0$.

Note that the cardinality of the finite set $\operatorname{Bx}(\sigma ; \beta)$ is given by the index of the sublattice generated by the generators of the cone $\sigma$ in $N$. If $\Im \beta$ is generic, the sets $\operatorname{Bx}(\sigma ; \beta)$ are disjoint, otherwise $\beta$ determines their intersections. When $\beta \in N \otimes \mathbb{R}$, the definition of $\operatorname{Bx}(\Sigma ; \beta)$ is equivalent to the definition of $\operatorname{Box}(\Sigma ; \beta)$ given earlier in this section, since in that case there is a one-to-one correspondence between the element $\alpha=\left(\alpha_{i}\right) \in \operatorname{Bx}(\sigma ; \beta)$ and the corresponding element $c=\sum_{i=1}^{k} \alpha_{i} v_{i} \in \sigma \cap(N+\beta)$. For a general $\beta \in N \otimes \mathbb{C}$, it may happen that for distinct elements $\alpha, \alpha^{\prime} \in \operatorname{Bx}(\Sigma ; \beta)$, we have that $\sum_{i=1}^{k} \alpha_{i} v_{i}=\sum_{i=1}^{k} \alpha_{i}^{\prime} v_{i}$. In order to account for such occurences, we have to adjust Definition 2.1 accordingly.

Definition 2.3. Given $\beta \in N \otimes \mathbb{C}$, the $R$-module $\mathbb{Z}[\Sigma ; \beta]$ is defined as the free abelian group with the basis elements $[n+\beta, \alpha]$ for all $n \in N$ and $\alpha=\left(\alpha_{i}\right) \in \operatorname{Bx}(\sigma ; \beta)$ for some maximal cone $\sigma$ of $\boldsymbol{\Sigma}$ such that

$$
n+\beta \in \sum_{i=1}^{k} \alpha_{i} v_{i}+\sum_{i \in I(\sigma)} \mathbb{Z}_{\geq 0} v_{i}
$$

The module structure is then defined by the product $\left[n^{\prime}\right] \cdot[n+\beta, \alpha]:=$ $\left[n^{\prime}+n+\beta, \alpha^{\prime}\right]$, if there exists a maximal cone $\sigma$ of $\boldsymbol{\Sigma}$ such that $n^{\prime} \in \sigma \cap N$, $\alpha \in \operatorname{Bx}(\sigma ; \beta)$, and

$$
n+\beta \in \sum_{i=1}^{k} \alpha_{i} v_{i}+\sum_{i \in I(\sigma)} \mathbb{Z}_{\geq 0} v_{i}, n^{\prime}+n+\beta \in \sum_{i=1}^{k} \alpha_{i}^{\prime} v_{i}+\sum_{i \in I(\sigma)} \mathbb{Z}_{\geq 0} v_{i} .
$$

If no such maximal cone $\sigma$ exists, then the product is 0 by definition.

If the product is non-zero, for any maximal cone $\sigma$ as above we have that $\alpha^{\prime} \in \operatorname{Bx}(\sigma ; \beta)$. It is easy to see that the module structure is well defined, i.e. the product structure does not depend on the choice of a maximal cone $\sigma$ in the above definition. 
Let $S_{\boldsymbol{\Sigma}}$ be the subring of $R$ generated by the elements $\left[v_{i}\right]$. We then have the the following direct sum decomposition of the $R$-module $\mathbb{Z}[\boldsymbol{\Sigma} ; \beta]$ into $S_{\boldsymbol{\Sigma}^{-}}$-submodules:

$$
\mathbb{Z}[\Sigma ; \beta]=\bigoplus_{\alpha \in \operatorname{Bx}(\boldsymbol{\Sigma} ; \beta)} S_{\boldsymbol{\Sigma}} \cdot\left[\sum_{i=1}^{k} \alpha_{i} v_{i}, \alpha\right]
$$

Remark 2.4. When $\beta \in N \otimes \mathbb{R}$, the $R$-module structures introduced in definitions 2.1 and 2.3 are identified under the map $[n+\beta] \mapsto[n+\beta, \alpha]$. Indeed, given $n+\beta \in \Sigma$, we consider the minimal cone $\sigma(n+\beta)$ of $\boldsymbol{\Sigma}$ containing $n+\beta$, and we write that $n+\beta=\sum_{i=1}^{k} q_{i} v_{i}$ with $q_{i} \in \mathbb{R}_{\geq 0}$, and $q_{i}=0$, if $i \notin I(\sigma(n+\beta))$. The element $\alpha=\left(\alpha_{i}\right) \in \operatorname{Bx}(\Sigma ; \beta) \subset \mathbb{R}^{k}$, is then uniquely defined by setting $\alpha_{i}:=\left\{q_{i}\right\}$, for all $i$.

As the following statement shows, the real and complex cases of the parameter $\beta$ are closer than one might have suspected at first glance.

Proposition 2.5. For any $\beta \in N \otimes \mathbb{C}$. there exists an isomorphism of $R$-modules $\mathbb{Z}[\Sigma ; \beta] \simeq \mathbb{Z}[\Sigma ; \Re \beta+\delta \Im \beta]$, for all sufficiently small $\delta>0$.

Proof. The combinatorics that determines the structure of $\mathbb{Z}[\Sigma ; \Re \beta+$ $\delta \Im \beta]$ as an $R$-module "stabilizes" when $\delta>0$ is sufficiently small. We must show that this stabilized $R$-module structure is isomorphic to the $R$-module $\mathbb{Z}[\Sigma ; \beta]$.

We define the map $\varphi_{\delta}: \mathbb{Z}[\boldsymbol{\Sigma} ; \beta] \rightarrow \mathbb{Z}[\boldsymbol{\Sigma} ; \Re \beta+\delta \Im \beta]$ by setting $\varphi_{\delta}([n+$ $\beta, \alpha]):=\left[n+\Re \beta+\delta \Im \beta, \alpha_{\delta}\right]$, where $\left(\alpha_{\delta}\right)_{i}:=\left\{\Re \alpha_{i}+\delta \Im \alpha_{i}\right\}$. Note that the map is well defined, since the support cone $\sigma\left(\alpha_{\delta}\right)$ is a subcone of $\sigma(\alpha)$, so any maximal cone which contains $\sigma(\alpha)$ will also contain $\sigma\left(\alpha_{\delta}\right)$. In fact, if $\delta>0$ is small enough so that $\Re \alpha_{i}+\delta \Im \alpha_{i}<1$ for all $i$, the support cones $\sigma(\alpha)$ and $\sigma\left(\alpha_{\delta}\right)$ coincide.

Let $n, n^{\prime} \in N$ and $\sigma$ a maximal cone such that

$$
n^{\prime} \in \sum_{i=1}^{k} \gamma_{i} v_{i}+\sum_{i \in I(\sigma)} \mathbb{Z}_{\geq 0} v_{i}, n+\beta \in \sum_{i=1}^{k} \alpha_{i} v_{i}+\sum_{i \in I(\sigma)} \mathbb{Z}_{\geq 0} v_{i}
$$

where $\gamma=\left(\gamma_{i}\right) \in \operatorname{Bx}(\Sigma ; 0) \subset \mathbb{Q}^{k}, \alpha=\left(\alpha_{i}\right) \in \operatorname{Bx}(\Sigma ; \beta) \subset \mathbb{C}^{k}$. In the $R$-module $\mathbb{Z}[\boldsymbol{\Sigma} ; \beta]$, we then have that $\left[n^{\prime}\right] \cdot[n+\beta, \alpha]=\left[n^{\prime}+n+\beta, \alpha^{\prime}\right]$, where $\alpha_{i}^{\prime}=\left\{\gamma_{i}+\Re \alpha_{i}\right\}+\sqrt{-1} \Im \alpha_{i}$, for all $i$. We see that

$$
\varphi_{\delta}\left(\left[n^{\prime}\right] \cdot[n+\beta, \alpha]\right)=\left[n^{\prime}+n+\Re \beta+\delta \Im \beta, \alpha_{\delta}^{\prime}\right]=\left[n^{\prime}\right] \cdot \varphi_{\delta}([n+\beta, \alpha]),
$$

where $\left(\alpha_{\delta}^{\prime}\right)_{i}=\left\{\gamma_{i}+\Re \alpha_{i}+\delta \Im \alpha_{i}\right\}$. This shows that $\varphi$ is an $R$-module homomorphism.

It is clear that $\varphi_{\delta}$ is a monomorphism. We now show that $\varphi_{\delta}$ is an epimorphism provided that $\delta>0$ is sufficiently small. Let $[n+\Re \beta+$ 
$\left.\delta \Im \beta, \alpha_{\delta}\right]$ be an element in the "stabilized" module $\mathbb{Z}[\Sigma ; \Re \beta+\delta \Im \beta]$. We assume that $\delta>0$ is small enough so that the support cone $\sigma\left(\alpha_{\delta}\right)$ is constant and contained in a maximal cone $\sigma$ such that

$$
n+\Re \beta+\delta \Im \beta=\sum_{i \in I(\sigma)}\left(\left(\alpha_{\delta}\right)_{i}+p_{i}\right) v_{i},
$$

with $p_{i} \in \mathbb{Z}_{\geq 0}$ independent of $\delta$. Since $n+\Re \beta \in \sigma$, there exists $\alpha \in$ $\operatorname{Bx}(\sigma ; \beta)$ such that

$$
n+\beta=\sum_{i \in I(\sigma)}\left(\alpha_{i}+p_{i}^{*}\right) v_{i},
$$

with $p_{i}^{*} \in \mathbb{Z}_{\geq 0}$. We are dealing with linear combinations over the generators of a maximal cone, so it must be the case that, for all $i$, $\left(\alpha_{\delta}\right)_{i}:=\left\{\Re \alpha_{i}+\delta \Im \alpha_{i}\right\}$. Hence, $\varphi_{\delta}([n+\beta, \alpha])=\left[n+\Re \beta+\delta \Im \beta, \alpha_{\delta}\right]$, and this ends the proof of the proposition.

Corollary 2.6. For any $\beta \in N \otimes \mathbb{C}$ and any sufficiently small $\delta>$ 0 , there exists a triple one-to-one correspondence $\alpha \leftrightarrow \alpha_{\delta} \leftrightarrow c\left(\alpha_{\delta}\right)$ among the finite sets $\mathrm{Bx}(\Sigma ; \beta) \subset \mathbb{C}^{k}, \operatorname{Bx}(\Sigma ; \Re \beta+\delta \Im \beta) \subset \mathbb{R}^{k}$ and $\operatorname{Box}(\Sigma ; \Re \beta+\delta \Im \beta) \subset N \otimes \mathbb{R}$, such that the support cones $\sigma(\alpha), \sigma\left(\alpha_{\delta}\right)$ and $\sigma\left(c\left(\alpha_{\delta}\right)\right)$ coincide.

The "pointwise" limit (in $\mathbb{R}^{k}$ ) of the set $\operatorname{Bx}(\Sigma ; \Re \beta+\delta \Im \beta)$ is closely related, but in general it is not equal to the set $\operatorname{Bx}(\Sigma ; \Re \beta)$. This observation is related to the issue discussed in Remark 2.2 .

We choose a basis $\left\{g_{1}, \ldots, g_{d}\right\}$ of the dual lattice $M=\operatorname{Hom}(N, \mathbb{Z})$, $d=\operatorname{rk} N$. Let $Z_{j} \in S_{\boldsymbol{\Sigma}} \subset \mathbb{Z}[\boldsymbol{\Sigma}]$ be given by

$$
Z_{j}:=\sum_{i=1}^{k} g_{j}\left(v_{i}\right)\left[v_{i}\right], 1 \leq j \leq d,
$$

and we denote by $Z:=\left(Z_{1}, \ldots, Z_{d}\right) \subset S_{\Sigma}$ the ideal generated by $Z_{1}, \ldots, Z_{d}$.

Definition 2.7. We call the quotient $R$-module

$$
H \mathbb{Z}(\Sigma ; \beta):=\mathbb{Z}[\Sigma ; \beta] / Z \cdot \mathbb{Z}[\Sigma ; \beta],
$$

the deformed $S R$-cohomology modules associated to the stacky fan $\Sigma$ and the element $\beta \in N \otimes \mathbb{C}$.

Of course, the constructions presented so far can be performed with the ring $\mathbb{Z}$ replaced by $\mathbb{Q}$ or $\mathbb{C}$. When $\beta \in N$, the deformed SRcohomology module coincides with the SR-cohomology ring $R / Z \cdot R$ which is the $\mathrm{SR}$-cohomology ring associated to the toric DM-stack $\mathbb{P}_{\boldsymbol{\Sigma}}$ 
as described by [5, Definition 3.1]. If in addition the stack is projective, then the SR-cohomology ring with $\mathbb{Q}$ coefficients is isomorphic to the orbifold cohomology ring of $\mathbb{P}_{\boldsymbol{\Sigma}}$ (cf. [3, Theorem 1.1]).

Since $Z_{j} \in S_{\boldsymbol{\Sigma}}$, the direct sum decomposition (3) descends to the following decomposition into a sum of $S_{\boldsymbol{\Sigma}} / Z$-submodules:

$$
H \mathbb{Z}(\Sigma ; \beta) \simeq \bigoplus_{\alpha \in \operatorname{Bx}(\boldsymbol{\Sigma} ; \beta)}\left(S_{\boldsymbol{\Sigma}} / Z\right) \cdot\left[\sum_{i=1}^{k} \alpha_{i} v_{i}, \alpha\right]
$$

We should also note that the ring $S_{\Sigma} / Z$ is isomorphic to the Chow ring of the toric Deligne-Mumford stack $\mathbb{P}_{\boldsymbol{\Sigma}}$ which is the same as the Chow ring of the toric variety $\mathbb{P}_{\Sigma}$, i.e. $A^{\star}\left(\mathbb{P}_{\Sigma}\right) \simeq A^{\star}\left(\mathbb{P}_{\Sigma}\right) \simeq S_{\Sigma} / Z$ (cf. [3, Lemma 5.1]).

\section{Deformations of the Grothendieck Ring of A REDUCED TORIC DM STACK}

Definition 3.1. The deformed Grothendieck ring $K \mathbb{C}(\boldsymbol{\Sigma} ; \beta)$ associated to the stacky fan $\Sigma=\left(\Sigma,\left\{v_{i}\right\}_{1 \leq i \leq k}\right)$ and the parameter $\beta \in N \otimes \mathbb{C}$ is defined to be the quotient of the Laurent polynomial ring $\mathbb{C}\left[R_{1}^{ \pm 1}, \ldots, R_{k}^{ \pm 1}\right]$ by the ideal $U+V$, where $U$ is the ideal generated by the relations

$$
\prod_{i \in I}\left(1-R_{i}\right)=0
$$

for any set $I \subset\{1, \ldots, k\}$ such that $v_{i}, i \in I$, do not generate a cone of $\Sigma$, and $V$ is the ideal generated by the relations

$$
\prod_{i=1}^{k} R_{i}^{f\left(v_{i}\right)}=e^{2 \pi \sqrt{-1} f(\beta)}
$$

for any linear function $f: N \rightarrow \mathbb{Z}$.

When $\beta \in N$, the definition can be given over $\mathbb{Z}$ and in that case, the ring is isomorphic to the Grothendieck ring $K_{0}\left(\mathbb{P}_{\boldsymbol{\Sigma}}\right)$ of the associated reduced toric Deligne-Mumford stack $\mathbb{P}_{\boldsymbol{\Sigma}}$ (cf. [5, Theorem 4.10]).

Proposition 3.2. The ring $K \mathbb{C}(\Sigma ; \beta)$ is Artinian. Its maximum ideals are in one-to-one correspondence with the elements of $\operatorname{Bx}(\Sigma ; \beta) \subset \mathbb{C}^{k}$ as follows: any element $\alpha=\left(\alpha_{i}\right)$ in $\operatorname{Bx}(\Sigma ; \beta)$ corresponds to the maximal ideal determined by the $n$-tuple $y=\left(y_{i}\right) \in \mathbb{C}^{k}$ with $y_{i}=e^{2 \pi \sqrt{-1} \alpha_{i}}$.

Proof. We need to solve for $y=\left(y_{i}\right) \in \mathbb{C}^{k}$ such that $\prod_{i \in I}\left(1-y_{i}\right)=0$, for any set $I \subset\{1, \ldots, k\}$ such that $\sigma(I)$ is not a cone in $\Sigma$, and $\prod_{i=1}^{k} y_{i}^{f\left(v_{i}\right)}=e^{2 \pi \sqrt{-1} f(\beta)}$ for all linear $f: N \rightarrow \mathbb{Z}$. 
Assume that $y=\left(y_{i}\right)$ is a solution. The first set of equations implies the existence of a maximal cone $\sigma$ such that $y_{1}=1$ for all $i \notin I(\sigma)$. The second set of equations shows that all $y_{i}$ are nonzero. There exists a unique choice $\left(\alpha_{i}\right) \in \mathbb{C}^{k}$ such that $y_{i}=e^{2 \pi \sqrt{-1}} \alpha_{i}$ and $0 \leq \Re \alpha_{i}<1$ if $i \in I(\sigma)$, and $\alpha_{i}=0$ if $i \notin I(\sigma)$. The second set of equations implies that $\sum_{i=1}^{k} \alpha_{i} v_{i}=n+\beta$, for some $n \in N$, so $\left(\alpha_{i}\right) \in \operatorname{Bx}(\Sigma ; \beta)$. Conversely, the definition of $\operatorname{Box}(\Sigma ; \beta)$ shows that, for any $\left(\alpha_{i}\right) \in \operatorname{Bx}(\Sigma ; \beta)$ the induced $y_{i}=e^{2 \pi \sqrt{-1}} \alpha_{i}$ provide a solution of the required system. It is easy to see that this is a one-to-one correspondence. It follows that the ring is indeed Artinian, since it is Noetherian of Krull dimension zero.

We denote by $(K \mathbb{C}(\Sigma ; \beta))_{\alpha}$ the localization of the Artinian ring $K \mathbb{C}(\Sigma ; \beta)$ at the maximal ideal corresponding to some $\alpha \in \operatorname{Bx}(\Sigma ; \beta)$. The Artinian ring $K \mathbb{C}(\Sigma ; \beta)$ is a direct sum of the Artinian local rings $(K \mathbb{C}(\Sigma ; \beta))_{\alpha}$. As the following result shows, this direct sum decomposition is the counterpart of the direct sum decomposition of the deformed cohomology $H \mathbb{Z}(\boldsymbol{\Sigma} ; \beta)$ into $S_{\boldsymbol{\Sigma}} / Z$-submodules as described by formula (4).

Let $S_{\boldsymbol{\Sigma}}(\alpha)$ denote the kernel of the $S_{\boldsymbol{\Sigma}^{-}}$module homomorphism $S_{\boldsymbol{\Sigma}} \rightarrow$ $\mathbb{Z}[\Sigma ; \beta]$ given by $[v] \mapsto[v] \cdot\left[\sum_{i=1}^{k} \alpha_{i} v_{i}, \alpha\right]$. We have that

$$
S_{\Sigma} / S_{\Sigma}(\alpha) \simeq S_{\Sigma} \cdot\left[\sum_{i=1}^{k} \alpha_{i} v_{i}, \alpha\right],
$$

as $S_{\boldsymbol{\Sigma}^{-}}$modules. In particular, we can write the following direct sum decomposition of deformed cohomology into $S_{\boldsymbol{\Sigma}} / Z$-submodules:

$$
H \mathbb{Z}(\Sigma ; \beta) \simeq \bigoplus_{\alpha \in \operatorname{Box}(\boldsymbol{\Sigma} ; \beta)} S_{\boldsymbol{\Sigma}} /\left(Z+S_{\boldsymbol{\Sigma}}(\alpha)\right)
$$

As we noted before, the ring $S_{\Sigma} / Z$ is isomorphic to the Chow ring of the toric Deligne-Mumford stack $A^{\star}\left(\mathbb{P}_{\boldsymbol{\Sigma}}\right)$.

Theorem 3.3. There exists a $\mathbb{C}$-vector space isomorphism

$$
\phi: K \mathbb{C}(\Sigma ; \beta) \simeq H \mathbb{C}(\Sigma ; \beta) .
$$

It is induced by $\mathbb{C}$-algebra isomorphisms

$$
\phi_{\alpha}(K \mathbb{C}(\Sigma ; \beta))_{\alpha} \simeq\left(S_{\Sigma} \otimes_{\mathbb{Z}} \mathbb{C}\right) /\left(Z+S_{\Sigma}(\alpha)\right),
$$

for each element $\alpha=\left(\alpha_{i}\right) \in \operatorname{Bx}(\Sigma ; \beta)$, induced by the identifications $R_{i}=e^{2 \pi \sqrt{-1}} \alpha_{i}+\left[v_{i}\right]$, for all $i, 1 \leq i \leq k$. 
Proof. Let us define the ring homomorphism $\phi_{\alpha}: \mathbb{C}\left[R_{1}^{ \pm 1}, \ldots, R_{k}^{ \pm 1}\right] \rightarrow$ $\left(S_{\boldsymbol{\Sigma}} \otimes_{\mathbb{Z}} \mathbb{C}\right) /\left(Z+S_{\boldsymbol{\Sigma}}(\alpha)\right)$ by setting

$$
\phi_{\alpha}(1):=1, \phi_{\alpha}\left(R_{i}^{ \pm 1}\right):=e^{ \pm\left(2 \pi \sqrt{-1} \alpha_{i}+\left[v_{i}\right]\right)} .
$$

Firstly, we check that $\phi_{\alpha}$ actually descends to a ring homomorphism $K \mathbb{C}(\Sigma ; \beta) \rightarrow\left(S_{\boldsymbol{\Sigma}} \otimes_{\mathbb{Z}} \mathbb{C}\right) /\left(Z+S_{\boldsymbol{\Sigma}}(\alpha)\right)$. For, if $f: N \rightarrow \mathbb{Z}$ is linear, then

$$
\phi_{\alpha}\left(\prod_{i=1}^{k} R_{i}^{f\left(v_{i}\right)}\right)=e^{\sum_{i=1}^{k}\left(2 \pi \sqrt{-1} \alpha_{i}+\left[v_{i}\right]\right) f\left(v_{i}\right)} .
$$

But $\sum_{i=1}^{k} f\left(v_{i}\right)\left[v_{i}\right]$ is an element of the ideal $Z$, and $\sum_{i=1}^{k} \alpha_{i} f\left(v_{i}\right)=$ $f(n+\beta)$, for some $n \in N$, hence $\phi_{\alpha}\left(\prod_{i=1}^{k} R_{i}^{f\left(v_{i}\right)}\right)=e^{2 \pi \sqrt{-1} f(\beta)}$, as required. Let $I$ be a subset of $\{1, \ldots, k\}$ such that the cone $\sigma(I)$ is not in $\Sigma$, and let $J$ denote the nonempty set $I \backslash I(\sigma(\alpha))$. We see that $\alpha_{i}=0$ for $i \in J$, so $\phi_{\alpha}\left(\prod_{i \in J}\left(1-R_{i}\right)\right)=\prod_{i \in J}\left(1-e^{\left[v_{i}\right]}\right)$. But the rays generated by the vectors $v_{i}, i \in J$, and the rays of the cone $\sigma(\alpha)$ do not generate a cone in $\Sigma$, so $\prod_{i \in J}\left[v_{i}\right] \cdot[\alpha]=0$ in $\mathbb{Z}[\Sigma ; \beta]$. Hence, $\phi_{\alpha}\left(\prod_{i \in I}\left(1-R_{i}\right)\right)=\phi_{\alpha}\left(\prod_{i \in J}\left(1-R_{i}\right)\right)=0$ in $\left(S_{\boldsymbol{\Sigma}} \otimes_{\mathbb{Z}} \mathbb{C}\right) /\left(Z+S_{\boldsymbol{\Sigma}}(\alpha)\right)$.

Secondly, we must check that $\phi_{\alpha}$ descends further to a ring homomorphism $\left(K \mathbb{C}(\Sigma ; \beta) \otimes_{\mathbb{Z}} \mathbb{C}\right)_{\alpha} \rightarrow\left(S_{\boldsymbol{\Sigma}} \otimes_{\mathbb{Z}} \mathbb{C}\right) /\left(Z+S_{\boldsymbol{\Sigma}}(\alpha)\right)$. We check this by showing that, for any Laurent polynomial $F\left(x_{1}^{ \pm 1}, \ldots, x_{k}^{ \pm 1}\right)$ with complex coefficients which is not in the maximal ideal of the localization, i.e. such that $F\left(e^{ \pm 2 \pi \sqrt{-1} \alpha_{1}}, \ldots, e^{ \pm 2 \pi \sqrt{-1} \alpha_{k}}\right) \neq 0$, it is true that $\phi_{\alpha}\left(F\left(R_{1}^{ \pm 1}, \ldots, R_{k}^{ \pm 1}\right)\right)$ is a unit in $\left(S_{\boldsymbol{\Sigma}} \otimes_{\mathbb{Z}} \mathbb{C}\right) /\left(Z+S_{\boldsymbol{\Sigma}}(\alpha)\right)$. But

$$
\phi_{\alpha}\left(F\left(R_{1}^{ \pm 1}, \ldots, R_{k}^{ \pm 1}\right)=F\left(e^{ \pm\left(2 \pi \sqrt{-1} \alpha_{1}+\left[v_{1}\right]\right)}, \ldots, e^{ \pm\left(2 \pi \sqrt{-1} \alpha_{k}+\left[v_{k}\right]\right)}\right) .\right.
$$

Note that the elements $\left[v_{i}\right]$ are nilpotent in the $\operatorname{ring} S_{\boldsymbol{\Sigma}} / Z$ since they represent classes in the Chow ring $A^{\star}\left(\mathbb{P}_{\boldsymbol{\Sigma}}\right) \simeq S_{\boldsymbol{\Sigma}} / Z$. Hence, the right hand side of the above equation is a polynomial with complex coefficients in the variables $\left[v_{i}\right]$ whose evaluation at $(0, \ldots, 0)$ is non-zero, so its inverse $1 / F\left(e^{ \pm\left(2 \pi \sqrt{-1} \alpha_{1}+\left[v_{1}\right]\right)}, \ldots, e^{ \pm\left(2 \pi \sqrt{-1} \alpha_{k}+\left[v_{k}\right]\right)}\right)$ is also a polynomial in the variables $\left[v_{i}\right]$, i.e. an element in $\left(S_{\boldsymbol{\Sigma}} \otimes_{\mathbb{Z}} \mathbb{C}\right) /\left(Z+S_{\boldsymbol{\Sigma}}(\alpha)\right)$. Therefore $\phi_{\alpha}:(K \mathbb{C}(\Sigma ; \beta))_{\alpha} \rightarrow S_{\boldsymbol{\Sigma}} /\left(Z+S_{\boldsymbol{\Sigma}}(\alpha)\right) \otimes_{\mathbb{Z}} \mathbb{C}$ is indeed a well defined ring homomorphism.

We now define a ring homomorphism $\psi_{\alpha}: S_{\boldsymbol{\Sigma}} \otimes_{\mathbb{Z}} \mathbb{C} \rightarrow(K \mathbb{C}(\boldsymbol{\Sigma} ; \beta))_{\alpha}$. Any element in the maximal ideal of an Artinian local ring is nilpotent. In particular, $e^{-2 \pi \sqrt{-1} \alpha_{i}} R_{i}$ is unipotent in $(K \mathbb{C}(\Sigma ; \beta))_{\alpha}$. We set

$$
\psi_{\alpha}(1):=1, \psi_{\alpha}\left(\left[v_{i}\right]\right):=\log \left(e^{-2 \pi \sqrt{-1} \alpha_{i}} R_{i}\right) .
$$

The definition uses the usual expansion of the log function given by $\log x=\sum_{j \geq 1}(-1)^{j+1}(x-1)^{j} / j$. 
For any linear function $f: N \rightarrow \mathbb{Z}$, we can see that

$$
\psi_{\alpha}\left(\sum_{i=1}^{k} f\left(v_{i}\right)\left[v_{i}\right]\right)=\log \left(e^{-2 \pi \sqrt{-1} \sum_{i=1}^{k} \alpha_{i} f\left(v_{i}\right)} \prod_{i=1}^{k} R_{i}^{f\left(v_{i}\right)}\right)=0
$$

since $\sum_{i=1}^{k} \alpha_{i} f\left(v_{i}\right)=f(n+\beta)$, for some $n \in N$, and $\prod_{i=1}^{k} R_{i}^{f\left(v_{i}\right)}=$ $e^{2 \pi \sqrt{-1} f(\beta)}$ in $(K \mathbb{C}(\Sigma ; \beta))_{\alpha}$. Let $v=\sum_{i \in I} p_{i} v_{i}, p_{i} \in \mathbb{Z}_{>0}$, be an element in $N$ such that $[v] \cdot\left[\sum_{i=1}^{k} \alpha_{i} v_{i}, \alpha\right]=0$ in $\mathbb{Z}[\Sigma ; \beta]$. From the definition of the product, we see that the rays of the cones $\sigma(I)$ and $\sigma(\alpha)$ do not generate a cone of the fan $\Sigma$. It is enough to show that the element $\prod_{i \in I}\left[v_{i}\right]$ gets mapped to zero. We can write that:

$$
\begin{aligned}
& \psi_{\alpha}\left(\prod_{i \in I}\left[v_{i}\right]\right)=\prod_{i \in I \cup I(\sigma(\alpha))}\left(1-R_{i}\right) \prod_{i \in I \backslash I(\sigma(\alpha))} \frac{\log R_{i}}{1-R_{i}} \\
& \cdot \prod_{i \in I \cap I(\sigma(\alpha))} \log \left(e^{-2 \pi \sqrt{-1} \alpha_{i}} R_{i}\right)\left(1-R_{i}\right)^{-1} \prod_{i \in I(\sigma(\alpha)) \backslash I}\left(1-R_{i}\right)^{-1} .
\end{aligned}
$$

The last three subfactors in the above product are all elements in the ring $(K \mathbb{C}(\Sigma ; \beta))_{\alpha}$, and, since the vectors $v_{i}$ with $i \in I \cup I(\sigma(\alpha))$ do not generate a cone of $\Sigma$, the first subfactor is zero. We have obtained that $\psi_{\alpha}([v])=\psi_{\alpha}\left(\prod_{i \in I}\left[v_{i}\right]\right)=0$, as required.

Hence, $\psi_{\alpha}$ descends to a morphism from $S_{\boldsymbol{\Sigma}} \otimes_{\mathbb{Z}} \mathbb{C} /\left(Z+S_{\boldsymbol{\Sigma}}(\alpha)\right)$ to $(K \mathbb{C}(\Sigma ; \beta))_{\alpha}$. It is clear that $\phi_{\alpha}$ and $\psi_{\alpha}$ are inverse maps, so the results follows.

Remark 3.4. Certainly, the isomorphism $\phi$ has the flavor of a Chern character. It would be interesting to make this assertion precise in a geometrically meaningful way. For a brief discussion of a similar issue, see [5, Remarks 5.4, 5.5].

Example 3.5. Consider the stacky fan $\Sigma$ in $\mathbb{Z}^{2}$ determined by the vectors $v_{1}=(1,0), v_{2}=(0,1), v_{3}=(-2,-1)$ and the obvious cones, and $\beta=(a, b) \in N \otimes \mathbb{C}$. The associated Deligne-Mumford stack is the stacky weighted projective space $\mathbb{P}(2,1,1)$.

According to Proposition $[3.2$, the maximum ideals of the $K \mathbb{C}(\Sigma ; \beta)$ by solving for $P=\left(y_{1}, y_{2}, y_{3}\right) \in\left(\mathbb{C}^{*}\right)^{3}$ the following system

$$
\left(1-y_{1}\right)\left(1-y_{2}\right)\left(1-y_{3}\right)=0, y_{1} y_{3}^{-2}=e^{2 \pi \sqrt{-1} a}, y_{2} y_{3}^{-1}=e^{2 \pi \sqrt{-1} b}
$$


We obtain four solutions $P_{i} \in\left(\mathbb{C}^{*}\right)^{3}$ which for generic values $a, b \in \mathbb{C}$ are distinct:

$$
\begin{aligned}
& P_{1}=\left(1, e^{2 \pi \sqrt{-1}(-a / 2+b)}, e^{2 \pi \sqrt{-1}(-a / 2)}\right), \\
& P_{2}=\left(1, e^{2 \pi \sqrt{-1}(-a / 2+b+1 / 2)}, e^{2 \pi \sqrt{-1}(-a / 2+1 / 2)}\right), \\
& P_{3}=\left(e^{2 \pi \sqrt{-1}(a-2 b)}, 1, e^{2 \pi \sqrt{-1}(-b)}\right), \\
& P_{4}=\left(e^{2 \pi \sqrt{-1} a}, e^{2 \pi \sqrt{-1} b}, 1\right) .
\end{aligned}
$$

Of course, this four points correspond to four possibly distinct elements in $\operatorname{Box}(\Sigma ; \beta)$.

For any generic $\beta$, the ring $K \mathbb{C}(\Sigma ; \beta)$ is semisimple of dimension equal to 4 , which is the normalized volume of the polytope determined by the vectors $v_{i}$. The non-generic values of $\beta$ are hyperplanes in $\mathbb{C}^{2}$ where "collisions" of some of the points $P_{i}$ occur.

For any $\beta=(a, b) \in \mathbb{C}^{2}$ contained in a hyperplane of the form $-a / 2+b \in \mathbb{Z}$, we have that $P_{1}=P_{3}$, while along the hyperplanes $-a / 2+b+1 / 2 \in \mathbb{Z}$, we have that $P_{2}=P_{3}$. For any $\beta=(a, b)$ contained in a hyperplane of the form $-a / 2 \in \mathbb{Z}$, we have that $P_{1}=P_{4}$, while along the hyperplanes $-a / 2+1 / 2 \in \mathbb{Z}$, we have that $P_{2}=P_{4}$. Moreover, for any $\beta=(a, b)$ in a hyperplane of the form $b \in \mathbb{Z}$, we have that $P_{3}=P_{4}$.

If we choose $\beta=(0,0)$, we have that $P_{1}=P_{3}=P_{4}$, and $K \mathbb{C}(\Sigma ; \beta) \cong$ $K_{0}(\mathbb{P}(2,1,1)) \otimes \mathbb{C} \simeq \mathbb{C}\left[R_{3}^{ \pm}\right] /\left(1-R_{3}\right)^{3}\left(1+R_{3}\right)$. For $\beta=(0,1 / 2)$, we have that $P_{1}=P_{4}, P_{2}=P_{3}$, and $K \mathbb{C}(\Sigma ; \beta) \cong \mathbb{C}\left[R_{3}^{ \pm}\right] /\left(1-R_{3}\right)^{2}\left(1+R_{3}\right)^{2}$. Of

course, in both cases we have that $R_{3}$ is the class corresponding to $v_{3}$.

\section{Application: Gamma series solutions to the Better BEHAVED GKZ HYPERGEOMETRIC SYSTEM WHEN $\beta \in N \otimes \mathbb{C}$}

As before, we assume that $\Sigma=\left(\Sigma,\left\{v_{i}\right\}_{1 \leq i \leq k}\right)$ is a simplicial stacky fan in the lattice $N$. In this section, $\mathcal{A}$ denotes the set $\left\{v_{1}, v_{2}, \ldots, v_{k}\right\} \subset$ $N$, and we allow for the possibility that some the elements of $\mathcal{A}$ do not generate rays of the fan $\boldsymbol{\Sigma}$. We denote by $I(\boldsymbol{\Sigma}) \subset\{1,2, \ldots k\}$, the set of indices $i$ such that $v_{i}$ generates a ray of $\Sigma$. We also assume that there exists a group homomorphism $\operatorname{deg}: N \rightarrow \mathbb{Z} \operatorname{such}$ that $\operatorname{deg}\left(v_{i}\right)=1$ for all $i, 1 \leq i \leq k$, and that the elements $v_{i}, 1 \leq i \leq k$, generate the lattice $N$ as a group. We assume that the fan $\Sigma$ is induced by a regular triangulation of the convex polytope $\Delta$, the convex hull of the vectors $v_{i}$. In particular, the support of the fan $\Sigma$ (denoted by $\Sigma$ so far in this note) coincides with the cone $K \subset N \otimes \mathbb{R}$ over the polytope $\Delta$ generated by the vectors $v_{i}$. We assume that the fixed parameter $\beta$ is in $N \otimes \mathbb{C}$. 
Definition 4.1. For any parameters $\chi, \xi \in N \otimes \mathbb{R}$, the shadow module $\mathbb{Z}[\boldsymbol{\Sigma} ; \chi]_{\xi}$ is defined as the $R$-submodule of $\mathbb{Z}[\Sigma ; \chi]$ generated by all $[n+\chi] \in \Sigma$, with $n \in N$, such that $n+\chi+\epsilon \xi \in \Sigma$ for all sufficiently small $\epsilon>0$.

The arguments used in [2, Section 3] essentially show that the following theorem holds:

Proposition 4.2. The ring $R=\mathbb{C}[\boldsymbol{\Sigma}]$, as well as the $R$-modules $\mathbb{C}[\Sigma ; \chi]$ and $\mathbb{C}[\Sigma ; \chi]_{\xi}$ are Cohen-Macaulay of dimension $d$. Moreover, for any basis $\left(g_{1}, \ldots, g_{d}\right)$ of $M=\operatorname{Hom}(N, \mathbb{Z})$, the elements

$$
Z_{j}=\sum_{i, i \in I(\boldsymbol{\Sigma})} g_{j}\left(v_{i}\right)\left[v_{i}\right]
$$

form a regular sequence in $R$ (and hence in $\mathbb{C}[\Sigma ; \chi]$ and $\mathbb{C}[\Sigma ; \chi]_{\xi}$ ).

Corollary 4.3. The quotients $H \mathbb{C}(\boldsymbol{\Sigma} ; 0)=\mathbb{C}[\boldsymbol{\Sigma}] / Z \cdot \mathbb{C}[\boldsymbol{\Sigma}], H \mathbb{C}(\boldsymbol{\Sigma} ; \chi)=$ $\mathbb{C}[\Sigma ; \chi] / Z \cdot \mathbb{C}[\Sigma ; \chi]$, and $\mathbb{C}[\Sigma ; \chi]_{\xi} / Z \cdot \mathbb{C}[\Sigma ; \chi]_{\xi}$, have dimension equal to the normalized volume of $\Delta$.

We now begin discussing the main theme of this section, namely the construction of $\Gamma$-series solutions to the better behaved GKZ system for $\beta \in N \otimes \mathbb{C}$.

Definition 4.4. For each $\alpha=\left(\alpha_{i}\right) \in \operatorname{Bx}(\Sigma ; \beta)$ and any $v \in K \cap N$, we define the set $L(\alpha, v) \subset \mathbb{C}^{n}$ of collections $l=\left(l_{i}\right)_{1 \leq i \leq k}$ such that

$$
\sum_{i=1}^{k} l_{i} v_{i}=\beta-v
$$

and $l_{i}-\alpha_{i}$ are integers for all $i, 1 \leq i \leq k$.

Since the elements $v_{i}, 1 \leq i \leq k$, generate the lattice $N$ as a group, we see that, for a given $v \in K$, any solution to the equation $\sum_{i=1}^{k} l_{i} v_{i}=$ $\beta-v$ such that $l_{i} \in \mathbb{Z}$ if $i \notin I(\sigma)$ for a maximal cone $\sigma$ in $\Sigma$, belongs to the set $L(\alpha, v)$ for some $\alpha \in \operatorname{Bx}(\sigma ; \beta)$.

We now choose a small enough $\delta>0$ such that the results of Proposition 2.5 and Corollary 2.6 hold. In particular, we have an $R$-module isomorphism between $\mathbb{C}[\Sigma ; \beta]$ and $\mathbb{C}[\Sigma ; \Re \beta+\delta \Im \beta]$, and an induced triple one-to-one correspondence $\alpha \leftrightarrow \alpha_{\delta} \leftrightarrow c\left(\alpha_{\delta}\right)$ among the sets $\operatorname{Bx}(\Sigma ; \beta) \subset \mathbb{C}^{k}, \operatorname{Bx}(\Sigma ; \Re \beta+\delta \Im \beta) \subset \mathbb{R}^{k}$ and $\operatorname{Box}(\Sigma ; \Re \beta+\delta \Im \beta) \subset N \otimes \mathbb{R}$ such that the corresponding support cones coincide: $\sigma(\alpha)=\sigma\left(\alpha_{\delta}\right)=$ $\sigma\left(c\left(\alpha_{\delta}\right)\right)$. In what follows, it will be convenient to use the notation

$$
\beta_{\delta}:=\Re \beta+\delta \Im \beta \in N \otimes \mathbb{R} .
$$


For a given $x=\left(x_{1}, \ldots, x_{k}\right)$ in $\left(\mathbb{C}^{\star}\right)^{k}$, and $v \in K \cap N$, we introduce the formal $\Gamma$-series $\Phi_{v}(x)$ with values in the completion $\mathbb{C}\left[\Sigma ; \beta_{\delta}\right]^{\wedge}$ of the graded $R$-module $\mathbb{C}\left[\Sigma ; \beta_{\delta}\right]$ as follows

$$
\Phi_{v}(x):=\sum_{c\left(\alpha_{\delta}\right) \in \operatorname{Box}\left(\Sigma ; \beta_{\delta}\right)} \sum_{l \in L(\alpha, v)} \prod_{i=1}^{k} \frac{x_{i}^{l_{i}+D_{i}}}{\Gamma\left(l_{i}+D_{i}+1\right)} \cdot\left[c\left(\alpha_{\delta}\right)\right],
$$

where

$$
D_{i}:=\left[v_{i}\right] \text { if } i \in I(\boldsymbol{\Sigma}) \text {, and } D_{i}:=0 \text {, otherwise, }
$$

and

$$
x_{i}^{l_{i}+D_{i}}:=e^{\left(l_{i}+D_{i}\right)\left(\log \left|x_{i}\right|+\sqrt{-1} \arg x_{i}\right)},
$$

for a choice of $\left(\arg x_{1}, \ldots, \arg x_{k}\right) \in \mathbb{R}^{k}$.

According to [6, Proposition 2.12], for each $v \in K \cap N$, the formal series $\Phi_{v}(x)$ induces a well defined map from a non-empty open set $U_{\Sigma}$ in $\mathbb{C}^{k}$ to the completion $\mathbb{C}\left[\Sigma, \beta_{\delta}\right]^{\wedge}$ of the graded ring $\mathbb{C}\left[\Sigma, \beta_{\delta}\right]$.

Lemma 4.5. i) For any $c\left(\alpha_{\delta}\right) \in \operatorname{Box}\left(\Sigma ; \beta_{\delta}\right), v \in \Sigma \cap N, l \in$ $L(\alpha, v)$ and $x \in \mathbb{C}^{k}$, the product

$$
\prod_{i=1}^{k} \frac{x_{i}^{l_{i}+D_{i}}}{\Gamma\left(l_{i}+D_{i}+1\right)} \cdot\left[c\left(\alpha_{\delta}\right)\right]
$$

belongs to the $R$-submodule $\mathbb{C}\left[\Sigma ; \beta_{\delta}\right]_{\Re \beta} \subset \mathbb{C}\left[\Sigma ; \beta_{\delta}\right]$.

ii) For any element $[w] \in \mathbb{C}\left[\Sigma ; \beta_{\delta}\right]_{\Re \beta}$, there exists a maximal cone $\sigma$ of $\Sigma$, a unique element $c\left(\alpha_{\delta}\right) \in \operatorname{Box}\left(\sigma ; \beta_{\delta}\right)$, and elements $v \in K \cap N, l=\left(l_{i}\right) \in L(\alpha, v)$, such that $l_{i}=0$ if $i \notin I(\sigma)$, and

$$
w \in c\left(\alpha_{\delta}\right)+\sum_{i, l_{i} \in \mathbb{Z}_{<0}} v_{i}+\sum_{i \in I(\sigma)} \mathbb{Z}_{\geq 0} v_{i} .
$$

Proof. i) Note first that the product is zero, unless there exists a maximal cone $\sigma$ such that $I\left(\sigma\left(\alpha_{\delta}\right)\right) \subset I(\sigma)$ and $I(\sigma)$ contains all $i$ with $l_{i} \in \mathbb{Z}_{<0}$. Let $\sigma$ be such a maximal cone. For any $i, i \notin I(\sigma)$, we have that $l_{i} \in \mathbb{Z}_{\geq 0}$.

The product lies in the the $R$-submodule of $\mathbb{C}\left[\Sigma ; \beta_{\delta}\right]$ generated by $[w]$ where

$$
w=\sum_{i=1}^{k}\left(\alpha_{\delta}\right)_{i} v_{i}+\sum_{i, l_{i} \in \mathbb{Z}_{<0}} v_{i}=n+\beta_{\delta},
$$

for some $n \in N$. Since $l_{i}-\alpha_{i}$ are integers for all $i$, and $\sigma(\alpha)=\sigma\left(\alpha_{\delta}\right)$, it is certainly the case that $l_{i} \in \mathbb{Z}$ if and only if $i \notin I\left(\sigma\left(\alpha_{\delta}\right)\right)$.

For any $\epsilon>0$ we can write that

$$
w+\epsilon(\Re \beta-v)=w+\epsilon \sum \Re l_{i} v_{i}
$$




$$
=\sum_{i, i \in I\left(\sigma\left(\alpha_{\delta}\right)\right)}\left(\left(\alpha_{\delta}\right)_{i}+\epsilon \Re l_{i}\right) v_{i}+\sum_{i, l_{i} \in \mathbb{Z}_{<0}}\left(1+\epsilon l_{i}\right) v_{i}+\sum_{i, l_{i} \in \mathbb{Z}_{\geq 0}} \epsilon l_{i} v_{i} .
$$

But $0<\left(\alpha_{\delta}\right)_{i}<1$ for any $i \in I\left(\sigma\left(\alpha_{\delta}\right)\right)$, so we indeed have that $w+\epsilon(\Re \beta-v)$ belongs to the cone $\Sigma$ for sufficiently small $\epsilon>0$. Since $v \in \Sigma$, we also have that $w+\epsilon \Re \beta \in \Sigma$, so $[w] \in \mathbb{C}\left[\Sigma ; \beta_{\delta}\right]_{\Re \beta}$.

ii) For any $[w] \in \mathbb{C}\left[\Sigma ; \beta_{\delta}\right]$, there exists $n \in N$ such that $w=n+\beta_{\delta}$. We choose a maximal cone $\sigma$ such that $w+\epsilon \beta_{\delta} \in \sigma$ for all sufficiently small $\epsilon>0$. As explained in Remark 2.4 since $\beta_{\delta} \in N \otimes \mathbb{R}$, there exists a uniquely defined $c\left(\alpha_{\delta}\right)=\sum_{i=1}^{k}\left(\alpha_{\delta}\right)_{i} v_{i} \in \operatorname{Box}(\Sigma ; \beta)$ contained in $\sigma$ such that

$$
w=n+\beta_{\delta}=c\left(\alpha_{\delta}\right)+\sum_{i \in I(\sigma)} p_{i} v_{i}=\sum_{i \in I(\sigma)}\left(\left(\alpha_{\delta}\right)_{i}+p_{i}\right) v_{i},
$$

with $p_{i} \in \mathbb{Z}_{\geq 0}$. Under the correspondence between $\alpha_{\delta} \in \operatorname{Bx}\left(\boldsymbol{\Sigma} ; \beta_{\delta}\right) \subset \mathbb{R}^{k}$ and $\alpha \in \operatorname{Bx}(\Sigma ; \beta) \subset \mathbb{C}^{k}$, we have that $\left(\alpha_{\delta}\right)_{i}=\left\{\Re \alpha_{i}+\delta \Im \alpha_{i}\right\}$. In particular, this means that

$$
n+\beta \in \sum_{i \in I(\sigma)}\left(\alpha_{i}+\mathbb{Z}\right) v_{i}
$$

For any $n \in N$, there exists a unique $v \in \operatorname{Box}(\sigma ; 0) \subset N$, such that $n \in-v+\sum_{i \in I(\sigma)} \mathbb{Z} v_{i}$. We conclude that we are able to write

$$
\beta-v=\sum_{i=1}^{k} l_{i} v_{i}
$$

with $l=\left(l_{i}\right) \in L(\alpha, v)$ and $l_{i}=0$ if $i \notin I(\sigma)$. By comparing this expression with equation (6), it is easy to see that $w+\epsilon \beta_{\delta} \in \sigma$ for all sufficiently small $\epsilon>0$, implies that $p_{i} \in \mathbb{Z}_{>0}$ for all $i$ with $l_{i} \in \mathbb{Z}_{<0}$, and this ends the proof of the lemma.

As the first part of this lemma indicates, the series $\Phi_{v}(x)$ takes values in the completion $\left(\mathbb{C}\left[\Sigma ; \beta_{\delta}\right]_{\Re \beta}\right)^{\wedge}$ of the graded $R$-module $\mathbb{C}\left[\Sigma ; \beta_{\delta}\right]_{\Re \beta}$.

Theorem 4.6. For any linear map

$$
h: \mathbb{C}\left[\Sigma ; \beta_{\delta}\right]_{\Re \beta} /\left(Z_{1}, \ldots, Z_{d}\right) \mathbb{C}\left[\Sigma ; \beta_{\delta}\right]_{\Re \beta} \rightarrow \mathbb{C},
$$

the sequence of functions $\left(h \circ \Phi_{v}\left(x_{1}, \ldots, x_{v}\right)\right)_{v \in K \cap N}$ satisfies the better behaved GKZ hypergeometric equations (11) and (2) on $U_{\Sigma}$ corresponding to the set $\mathcal{A}$ and parameter $\beta \in N \otimes \mathbb{C}$.

Proof. For the set of equations (1), note that taking the $j$-th partial derivative of the summand over $\left(l_{i}\right)_{1 \leq i \leq k} \in L(\alpha, v)$ in the series $\Phi_{v}$, 
replaces it by a summand over $\left(l_{i}-\delta_{j}^{i}\right)_{1 \leq i \leq k}$. It is then enough to note that

$$
L(\alpha, v)-\left(\delta_{j}^{i}\right)_{1 \leq i \leq k}=L\left(\alpha, v+v_{j}\right) .
$$

For the set of equations (2), our convention that $D_{j}=0$ if $j \notin I(\boldsymbol{\Sigma})$, implies that

$$
\sum_{i=1}^{k} g\left(v_{i}\right) D_{i}=\sum_{i, i \in I(\boldsymbol{\Sigma})} g\left(v_{i}\right) D_{i}
$$

for any $g \in M=\operatorname{Hom}(N, \mathbb{Z})$. Hence

$$
\left(-g(\beta-v)+\sum_{i=1}^{k} g\left(v_{i}\right) x_{i} \partial_{i}\right) \Phi_{v}=\left(\sum_{i=1}^{k} g\left(v_{i}\right) D_{i}\right) \Phi_{v} .
$$

The previous lemma shows that the series $\Phi_{v}$ takes values in $\left.\mathbb{C}\left[\Sigma ; \beta_{\delta}\right]\right]_{\Re \beta}$, so the result follows after we observe that $\sum_{i=1}^{k} g\left(v_{i}\right) D_{i}$ is a linear combination of the $Z_{j}$ 's.

Accordingly, it is convenient to view each $\Gamma$-series $\Psi_{v}(x), v \in K \cap$ $N$, as a map from the non-empty open set $U_{\boldsymbol{\Sigma}}$ in $\mathbb{C}^{k}$ to the finite dimensional vector space $\mathbb{C}\left[\Sigma ; \beta_{\delta}\right]_{\Re \beta} /\left(Z_{1}, \ldots, Z_{d}\right) \mathbb{C}\left[\Sigma ; \beta_{\delta}\right]_{\Re \beta}$. We now prove the required linear independence result.

Proposition 4.7. If $h: \mathbb{C}\left[\Sigma ; \beta_{\delta}\right]_{\Re \beta} /\left(Z_{1}, \ldots, Z_{d}\right) \mathbb{C}\left[\Sigma ; \beta_{\delta}\right]_{\Re \beta} \rightarrow \mathbb{C}$ is a linear map such that $h \circ \Phi_{v}(x)=0$, for all $v \in K \cap N$ and any $x \in U_{\boldsymbol{\Sigma}}$, then $h=0$.

Proof. The proof is very similar to the proof of [6, Proposition 2.19]. We include it here since the context and the notation are slightly changed. Assume that there exists some $x \in \mathbb{C}\left[\Sigma ; \beta_{\delta}\right]_{\Re \beta} /\left(Z_{1}, \ldots, Z_{d}\right) \mathbb{C}\left[\Sigma ; \beta_{\delta}\right]_{\Re \beta}$ such that $h(x) \neq 0$. Let $P$ be the largest degree of such an element. Furthermore, we choose a representative $[w] \bmod Z \mathbb{C}\left[\Sigma ; \beta_{\delta}\right]_{\Re \beta}$ for $[x]$. According to part $i i$ ) of Lemma 4.5, there exists a maximal cone $\sigma$ and a unique $c\left(\alpha_{\delta}\right) \in \operatorname{Box}\left(\sigma ; \beta_{\delta}\right)$ an element $v \in K \cap N$, and a relation $l \in L(\alpha, v)$ such that

$$
w=c\left(\alpha_{\delta}\right)+\sum_{i, l_{i} \in \mathbb{Z}_{<0}} v_{i}+\sum_{i \in I(\sigma)} n_{i} v_{i}
$$

with $n_{i} \in \mathbb{Z}_{\geq 0}$.

For each $i$ such that $v_{i}$ with $i \in I(\boldsymbol{\Sigma})$, consider the loop of the form $x_{i}(t)=\epsilon \exp (2 \pi \sqrt{-1} t), x_{j}(t)=\epsilon, j \neq i, 0 \leq t \leq 1$, with $\epsilon>0$ a small real positive number. The action of the induced monodromy operator $T_{i}$ on the $\Gamma$-series $\Phi_{v}$ is given by $\exp \left(D_{i}\right)$. Since $D_{i}$ is nilpotent in $\mathbb{C}\left[\Sigma ; \beta_{\delta}\right]_{\Re \beta} / Z \cdot \mathbb{C}\left[\Sigma ; \beta_{\delta}\right]_{\Re \beta}$, there is a polynomial $g\left(T_{i}\right)$ such that 
$g\left(T_{i}\right) \Phi_{v}=D_{i} \Phi_{v}$, for every $i \in I(\boldsymbol{\Sigma})$. Hence

$$
\prod_{i} g\left(T_{i}\right)^{n_{i}} \Phi_{v}(x)=\prod_{i} D_{i}^{n_{i}} \Phi_{v}(x) .
$$

Since $h \circ \Phi_{v}(x)=0$ and we have analytically continued $\Phi_{v}$, we also have that

$$
h\left(\prod_{i} D_{i}^{n_{i}} \Phi_{v}(x)\right)=0 .
$$

The definition of the $\Gamma$-series $\Phi_{v}(x)$ and the fact that $D_{i}=\left[v_{i}\right]$ are nilpotent in $\mathbb{C}\left[\Sigma ; \beta_{\delta}\right]_{\Re \beta} / Z \cdot \mathbb{C}\left[\Sigma ; \beta_{\delta}\right]_{\Re \beta}$ shows that any induced GKZ solution can be written as the product of a monomial in the variables $x_{i}$ and an element of $\mathbb{C}\left[u_{j}^{-1}, \log u_{j}\right]\left[\left[u_{j}\right]\right]$ where $u_{j}, 1 \leq j \leq n-\operatorname{rank} N$, invariant variables under the action of the character torus $\operatorname{Hom}\left(L, \mathbb{C}^{\times}\right)$, where $L$ is the lattice of integral relations $\left(l_{i}\right)$ such that $\sum_{i=1}^{k} l_{i} v_{i}=0$.

Hence, in order to obtain the contradiction it is enough to show that, for $l \in L(\alpha, v)$ used in formula (7), the Fourier coefficient of $x^{l}=\prod x_{i}^{l_{i}}$ in the expansion of $h\left(\prod_{i} D_{i}^{n_{i}} \Phi_{v}(x)\right)$ is non-zero. Indeed, this coefficient is given by

$$
h\left(\prod D_{i}^{n_{i}} \cdot\left[c\left(\alpha_{\delta}\right)\right] \cdot \prod \frac{1}{\Gamma\left(l_{i}+D_{i}+1\right)}\right) .
$$

Notice that the terms that occur in the expansion of the expression in the argument of $h$ have degree at least $P$, while $[w]$ is the only element of that degree that occurs and its coefficient is nonzero. Since $h([w]) \neq 0$, the maximal property of $P$ implies that the coefficient of $x^{l}$ in the expansion of $h\left(\prod_{i} D_{i}^{n_{i}} \Phi_{v}(x)\right)$ is indeed non-zero. This ends the proof of the linear independence result.

According to [4, Corollary 3.9], the dimension of the space of solutions to the better behaved GKZ system is exactly $\operatorname{vol}(\Delta)$, so we conclude that the formal $\Gamma$-series produces the expected number of linearly independent analytic solutions to the better behaved GKZ system. More precisely, we have constructed the following "topological mirror symmetry" map:

Theorem 4.8. The map

$$
\begin{aligned}
\left(\mathbb{C}\left[\Sigma ; \beta_{\delta}\right]_{\Re \beta} / Z \cdot \mathbb{C}\left[\Sigma ; \beta_{\delta}\right]_{\Re \beta}\right)^{\vee} & \rightarrow \operatorname{Sol}\left(U_{\boldsymbol{\Sigma}}\right) \\
f & \rightarrow\left(f \circ \Phi_{v}\right)_{v \in K \cap N}
\end{aligned}
$$

produces a complete system of $\operatorname{vol}(\Delta)$ linearly independent solutions to the better behaved $G K Z$ system which are analytic in $U_{\boldsymbol{\Sigma}}$.

Remark 4.9. The result of this theorem should be compared with the results of [6, Corollary 2.21] which employ the "leading term module" 
$M(\beta)$ associated to the usual GKZ system for $\beta \in N$. The modules $M(\beta)$ are useful algebraic tools defined in loc. cit., but the dimensions of $M(\beta) / Z M(\beta)$ are quite hard to control for general values of $\beta$. In contrast, the shadow modules $\mathbb{C}\left[\Sigma ; \beta_{\delta}\right]_{\Re \beta}$ are maximal CohenMacaulay. It is possible to show that, for $\beta \in N$, we have that $\mathbb{C}[\Sigma ; \beta]_{\beta}=\sum_{v \in K \cap N} M(\beta-v)$.

Remark 4.10. It is possible to give a $K$-theoretic combinatorial description in the spirit of the discussion from Section 3 of the shadow modules $\mathbb{C}\left[\Sigma ; \beta_{\delta}\right]_{\Re \beta} / Z \cdot \mathbb{C}\left[\Sigma ; \beta_{\delta}\right]_{\Re \beta}$. Somewhat surprisingly, in our approach such a description is related to the notion of a $\gamma$-deformed version of the set $\operatorname{Box}(\Sigma ; \beta)$ mentioned in Remark 2.2 which we do not pursue in this note. It suffices to say that combining such a description with the tools developed in [6. Section 3] provides a $K$-theoretical version of the topological mirror symmetry map described above.

\section{ACKNOWLEDGEMENTS}

I would like to thank Lev Borisov for very useful comments and discussions. Many of the ideas presented in this note have been developed in the course of our joint collaboration. I would like to thank the organizers of the Maximilian Kreuzer Memorial Conference for the opportunity to speak at the conference in June 2011, and for the invitation to submit a contribution to this volume. This work is dedicated to the memory of Maximilian Kreuzer whose irresistible enthusiasm for subjects at the interface of string theory and algebraic geometry remains a great source of inspiration. The topics of this note, in particular toric geometry and GKZ systems, are among those which benefited greatly from his ideas.

\section{REFERENCES}

[1] V. V. Batyrev, Variations of mixed Hodge structure of affine hypersurfaces in algebraic tori, Duke Math. J. 69 (1993), 349-409.

[2] L. A. Borisov, String cohomology of a toroidal singularity, J. Algebraic Geom. 9 (2000), no. 2, 289-300, math.AG/9802052.

[3] L. A. Borisov, L. Chen, G. Smith, The orbifold Chow ring of toric Deligne-Mumford stacks, J. Amer. Math. Soc. 18 (2005), no. 1, 193-215, math. AG/0309229.

[4] L. A. Borisov, R. P. Horja, On the better behaved version of the GKZ hypergeometric system, preprint, 2010; arXiv:1011.5720 [math.AG].

[5] L. A. Borisov, R. P. Horja, On the $K$-theory of smooth toric DM stacks, Snowbird lectures on string geometry, 21-42, Contemp. Math., 401, Amer. Math. Soc., Providence, RI, 2006, math.AG/0503277.

[6] L. A. Borisov, R. P. Horja, Mellin-Barnes integrals as Fourier-Mukai transforms, Adv. Math. 207 (2006), no. 2, 876-927, math.AG/0510486. 
[7] I. M. Gelfand, M. M. Kapranov, A. V. Zelevinsky, Hypergeometric functions and toric varieties, (Russian) Funktsional. Anal. i Prilozhen. 23 (1989), no. 2, 12-26; translation in Funct. Anal. Appl. 23 (1989), no. 2, 94-106.

[8] S. Hosono, B.H. Lian, S.-T. Yau, Maximal degeneracy points of GKZ systems, J. Amer. Math. Soc. 10 (1997), no. 2, 427-443; alg-geom/9603014.

[9] L. F. Matusevich, E. Miller, U. Walther, Homological methods for hypergeometric families, J. Amer. Math. Soc. 18 (2005), no. 4, 919-941; math.AG/0406383.

[10] M. Saito, B. Sturmfels, N. Takayama, Gröbner deformations of hypergeometric differential equations, Springer, 2000.

[11] J. Stienstra, Resonant hypergeometric systems and mirror symmetry, Integrable systems and algebraic geometry (Kobe/Kyoto, 1997), 412-452, World Sci. Publishing, River Edge, NJ, 1998, math.AG/9711002.

Department of Mathematics, Oklahoma State University, StillwaTER, OK 74078, USA, HORJA@MATH.OKSTATE.EDU 